\title{
The Herschel-SPIRE photometer data processing pipeline
}

\section{Matt Griffin, C. Darren Dowell, Tanya Lim, George Bendo, Jamie Bock, et al.}

Matt Griffin, C. Darren Dowell, Tanya Lim, George Bendo, Jamie Bock, Christophe Cara, Nieves Castro-Rodriguez, Pierre Chanial, Dave Clements, Rene Gastaud, Steve Guest, Jason Glenn, Victor Hristov, Ken King, Glenn Laurent, Nanyao Lu, Gabrielle Mainetti, Huw Morris, Hien Nguyen, Pasquale Panuzzo, Chris Pearson, Frederic Pinsard, Michael Pohlen, Edward Polehampton, Davide Rizzo, Bernhard Schulz, Arnold Schwartz, Bruce Sibthorpe, Bruce Swinyard, Kevin Xu, Lijun Zhang, "The Herschel-SPIRE photometer data processing pipeline," Proc. SPIE 7010, Space Telescopes and Instrumentation 2008: Optical, Infrared, and Millimeter, 70102Q (12 July 2008); doi: 10.1117/12.788431

Event: SPIE Astronomical Telescopes + Instrumentation, 2008, Marseille, France 


\title{
The Herschel-SPIRE Photometer Data Processing Pipeline
}

\author{
Matt Griffin ${ }^{*}, \mathrm{a}$, C. Darren Dowell ${ }^{\mathrm{b}}$, Tanya Lim$^{\mathrm{c}}$, George Bendo ${ }^{\mathrm{d}}$, Jamie Bock ${ }^{\mathrm{b}}$, Christophe Cara ${ }^{\mathrm{e}}$, \\ Nieves Castro-Rodriguez ${ }^{\mathrm{f}}$, Pierre Chanial ${ }^{\mathrm{d}}$, Dave Clements ${ }^{\mathrm{d}}$, Rene Gastaud ${ }^{\mathrm{e}}$, Steve Guest ${ }^{\mathrm{c}}$, \\ Jason Glenn ${ }^{\mathrm{g}}$, Victor Hristov ${ }^{\mathrm{h}}$, Ken King ${ }^{\mathrm{c}}$, Glenn Laurent ${ }^{\mathrm{g}}$, Nanyao Lu $^{\mathrm{i}}$, Gabrielle Mainetti ${ }^{\mathrm{j}}$, \\ Huw Morris ${ }^{c}$, Hien Nguyen ${ }^{\mathrm{b}}$, Pasquale Panuzzo ${ }^{\mathrm{e}}$, Chris Pearson ${ }^{\mathrm{c}}$, Frederic Pinsard ${ }^{\mathrm{e}}$, \\ Michael Pohlen ${ }^{\mathrm{a}}$, Edward Polehampton ${ }^{\mathrm{c}, \mathrm{k}}$, Davide Rizzo ${ }^{\mathrm{d}}$, Bernhard Schulz ${ }^{\mathrm{i}}$, Arnold Schwartz, \\ Bruce Sibthorpe ${ }^{1}$, Bruce Swinyard ${ }^{\mathrm{c}}$, Kevin Xu' ${ }^{\mathrm{i}}$, and Lijun Zhang ${ }^{\mathrm{i}}$

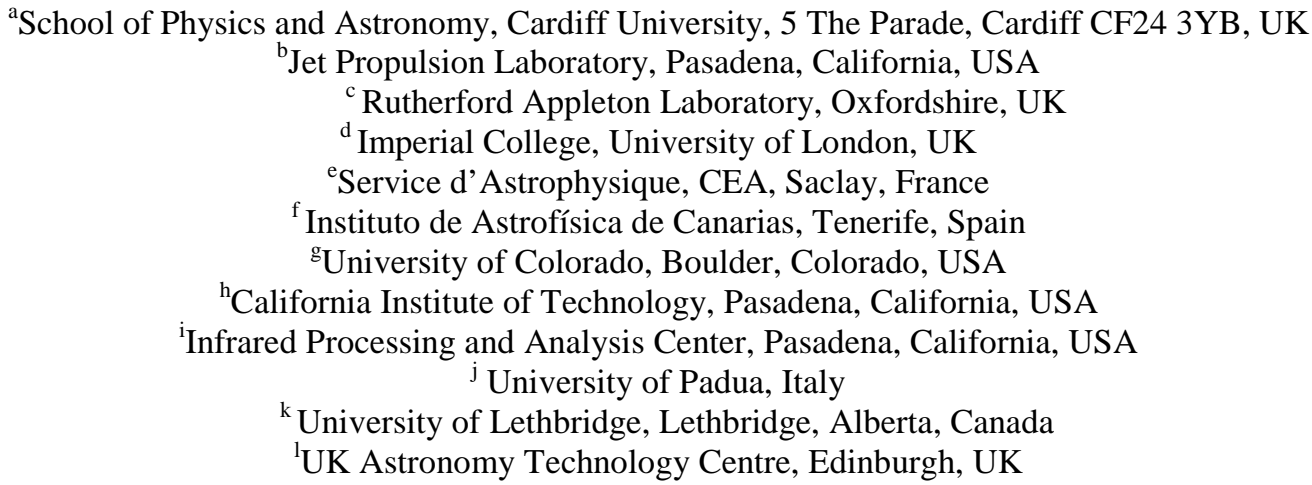

\begin{abstract}
We describe the on-board electronics chain and the on-ground data processing pipeline that will operate on data from the Herschel-SPIRE photometer to produce calibrated astronomical products. Data from the three photometer arrays will be conditioned and digitised by on-board electronics and sent to the ground with no further on-board data processing. On the ground, the data pipeline will process the data from point source, jiggle-map, and scan-map observations in a fully automatic manner, producing measured flux densities (for point source observations) or maps. It includes calculation of the bolometer voltages from the raw telemetry, glitch removal, and corrections for various effects including time constants associated with the detectors and electronics, electrical and optical crosstalk, detector temperature drifts, flatfielding, and non-linear response of the bolometers to strong sources. Flux density calibration will be with respect to standard astronomical sources with the planets Uranus and Neptune being adopted as the baseline primary standards. The pipeline will compute estimated values of in-beam flux density for a standard flat $v S(v)$ source spectrum.
\end{abstract}

Keywords: Herschel, Far Infrared, Submillimetre, Instrumentation, Data Processing

*E-mail: matt.griffin@astro.cf.ac.uk; Telephone: +44-(0)29-2087-4203; Fax: +44-(0)29-2087-4056

\section{INTRODUCTION}

The Spectral and Photometric Imaging Receiver (SPIRE) ${ }^{1}$ is one of three scientific instruments to fly on the European Space Agency's Herschel Space Observatory ${ }^{2}$. SPIRE contains a three-band imaging photometer and an imaging Fourier Transform Spectrometer (FTS). All SPIRE observations will be processed by pipelines operating fully automatically, which shall produce standard calibrated data products (maps, point source fluxes, spectral data cubes, etc.) with instrument artefacts removed. It will also be possible for users to run the pipelines interactively in order to modify parameters and assumptions if desired. In this paper we describe the pipeline processes to be used for the standard photometer observing modes: point source photometry, jiggle mapping (for small maps) and scan mapping (for large maps). The pipeline for the FTS is described by Fulton et al. ${ }^{3}$ 
The photometer pipelines are designed to be modular and flexible, and the algorithmic content of modules has been made as simple as possible, with the associated calibration files containing key parameters, many of which we expect to be updated and refined in flight. Because pipeline processing must be carried out automatically for all observations, no $a$ priori assumptions are made about the spatial distribution of the sky brightness distribution, except for point source photometry observations, in which case the fitting of flux density and position assumes that the source is point-like.

\section{PHOTOMETER OVERVIEW AND OBSERVING MODES}

\subsection{Photometer overview}

Three ${ }^{3} \mathrm{He}$-cooled bolometer arrays are used for broad-band photometry $(\lambda / \Delta \lambda \sim 3)$ in spectral bands centred on approximately 250,350 and $500 \mu \mathrm{m}$. The same $4 \times 8$ arcmin. field of view is observed simultaneously in the three bands through the use of two fixed dichroic beam-splitters. Signal modulation can be provided either by SPIRE's two-axis Beam Steering Mirror (BSM), or by scanning the telescope across the sky. An internal thermal source is available to provide a repeatable calibration signal for the detectors. There are three SPIRE warm electronics units: the Detector Control Unit (DCU) provides the bias and signal conditioning for the arrays and cold electronics, and demodulates and digitises the detector signals; the FPU Control Unit (FCU) controls the ${ }^{3} \mathrm{He}$ cooler and the two FPU mechanisms, and reads out all the FPU thermometers; and the Digital Processing Unit (DPU) runs the on-board software and interfaces with the spacecraft for commanding and telemetry. The $130 \mathrm{kbs}$ available data rate allows all detectors to be sampled and the data transmitted to the ground with no on-board processing.

The photometer detectors are designed to have first-order time constants of typically $6 \mathrm{~ms}$, and this has been verified in unit-level and instrument-level testing of the arrays. There is evidence from measurements on similar detectors (used in the Planck-HFI and BICEP instruments) that SPIRE-like bolometers may not exhibit a pure first-order response (characterised by a single time constant), but can also have a low-level slow response, with variation of detector responsivity with signal frequency, $\omega_{s}$, characterised by a transfer function of the following form:

$$
H\left(\omega_{\mathrm{s}}\right)=\frac{1-a}{1+j \omega_{\mathrm{s}} \tau_{1}}+\frac{a}{1+j \omega_{\mathrm{s}} \tau_{2}} .
$$

In extreme cases, the "slow response" time constant, $\tau_{2}$, can be several hundred ms, with the amplitude parameter $a$ up to $30 \%$. The detailed slow response characteristics of the SPIRE flight model detectors have not been measured on the ground, although no strong evidence of such effects is evident in test data. Measurements will be made during the Herschel Performance Verification phase involving scanning point sources at various speeds to characterise any slow response and determine the parameters $\tau_{2}$ and $a$; and the pipeline includes a module to correct for any slow component.

\subsection{Observing modes}

The SPIRE detectors are feedhorn-coupled, with $2 F \lambda$ horn entrance apertures for maximum aperture efficiency. This means that the diffraction-limited beams on the sky are separated by approximately twice the beam FWHM, requiring "jiggling" of the field of view using the BSM or scanning of the arrays across the sky to produce a fully sampled image. The photometer will have three principal observing modes, described in [1] and [4] and briefly summarised here.

Point or compact source photometry: The standard observing mode for point sources will use two particular sets of three detectors with overlapping beams on the sky in order to take data in the three bands simultaneously. The beam steering mirror will be used to make a seven-point map in which the nominal position and six hexagonally arranged neighbouring positions with an angular offset of 6" (approx. 1/3 of the smallest beam) will be observed in turn, with the central position observed twice, at the beginning of and the end of the sequence. Due to small asymmetries in the optical system, the ambient background power in the two chop positions will be slightly different. In order to subtract the resulting offset, the telescope will be nodded in the standard manner adopted on ground-based telescopes. The data will be fitted with a 2-D beam profile to determine the peak flux density and positional offset from the telescope pointed position, assuring that pointing or source position errors do not result in flux density errors. The data from all detectors in all of the arrays will also be available, providing sparsely sampled maps of the field around the object. The standard point source observation involves an A-B-B-A sequence where A and B are the two telescope nod positions. The onsource integration time for each of the four steps is 64 seconds, comprising eight seconds in each of the eight map positions. With the standard 2-Hz chop frequency, each of these eight-second sequences comprises 16 chop cycles. 
Jiggle mapping: This mode is similar to the standard jiggle-map observing mode used with SCUBA on the James Clerk Maxwell Telescope 5 . For mapping of regions up to few arcmin. in size, the BSM will perform a 64-point jiggle map, needed to achieve full spatial sampling in all bands simultaneously, with a step size of 9" (half-beam spacing at $250 \mu \mathrm{m}$ ). The jiggle-map field size is $4 \times 4$ arcmin. as the 2 -arcmin. regions at each end of the array will be chopped outside the field of view admitted by the photometer optics. Nodding is carried out as for point source observations. The nominal jiggle map observation incorporates four nod cycles, each covering 16 different jiggle positions.

In point source and jiggle-map mode, detector sampling is in synchronism with the BSM movements.

Scan mapping: For large maps (bigger than the SPIRE field of view), the telescope will be scanned across the sky at either 30" s $\mathrm{s}^{-1}$ (nominal) or $60^{\prime} \mathrm{s}^{-1}$ (fast). To give the beam overlap needed for full spatial sampling over a strip defined by one scan line, and to provide a uniform distribution of integration time over the area covered by the scan, the scan angle is tilted with respect to the array axes, and cross-scanning is implemented in order to minimise the effect of $1 / f$ drifts. During scan-map observations, the detectors will be sampled at $16 \mathrm{~Hz}$.

\section{ON-BOARD SIGNAL CHAIN}

The essential features of the bolometer bias and readout circuit are shown in Figure 1. The bolometers are AC sine-wave biased at a frequency adjustable in the range $50-300 \mathrm{~Hz}$, much faster than the bolometer thermal time constant, so that bias itself does not produce a temperature modulation. The bias is applied symmetrically via the two load resistors $\left(R_{\mathrm{L}} / 2\right.$ $\approx 8 \mathrm{M} \Omega$ each). The bolometer resistance at the operating point, $R_{\mathrm{b}}$, is typically $3 \mathrm{M} \Omega$. The $\mathrm{AC}$ bias up-converts the signal information to the bias frequency, getting well above the $1 / f$ noise knee of the JFET readout amplifiers. Each detector signal is fed to a JFET pair whose outputs are connected via a long cryoharness to the warm electronics in the Herschel Service Module.

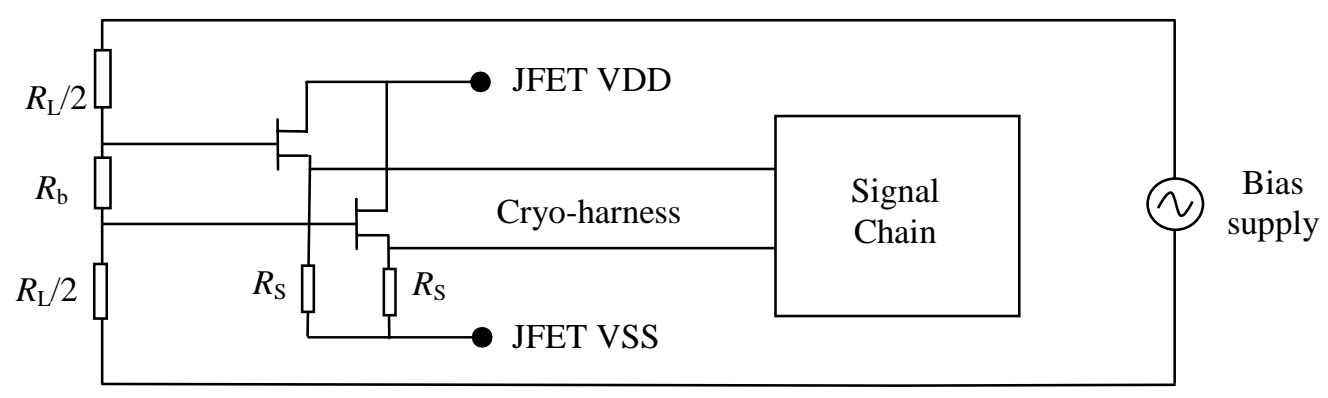

Figure 1: SPIRE bolometer bias and readout circuit.

The JFETs act as source followers, with a gain of typically 0.96 . The approx. $200 \mathrm{~mm}$ length of cryoharness between the detectors and the JFET modules (located outside the cold instrument enclosure) has a capacitance of typically $50 \mathrm{pf}$. This forms an RC circuit with the combined impedance of the bolometer and load resistors, which causes small amplitude and phase changes to the bolometer signals at the JFETs. These are corrected in the first stage of the pipeline.

Figure 2 is a block diagram of the analogue signal chain. The detector voltage signals are de-modulated by individual lock-in amplifiers (LIAs). An LIA comprises (i) a band-pass filter/amplifier to remove the DC component and amplify the signal; (ii) a square-wave synchronous demodulator which rectifies the signal; (iii) a low-pass filter which produces a low-frequency output proportional to the detector voltage. The output of the LIA is nominally a DC voltage proportional to the rms value of the voltage at the bolometer output. The LIA outputs are multiplexed and sampled for telemetry to the ground. In order to achieve the necessary 20-bit sampling using a 16-bit ADC (the highest resolution available with space qualified devices), an offset subtraction scheme is implemented, whereby an offset voltage is generated by a 4-bit DAC and subtracted from the detector voltage before the final stage of amplification prior to digitisation. The combination of the offset value and the ADC sample allows the bolometer voltage to be reconstructed on the ground to 20-bit accuracy.

Bandpass filter/amplifier: The bandpass filter/amplifier has 3-dB frequencies of $33 \mathrm{~Hz}$ and $1.3 \mathrm{KHz}$ and passes the $50-300 \mathrm{~Hz}$ range of allowed bias frequencies with gain between 220 and 260. 
Demodulator: The demodulator multiplies the alternating input signal by a square wave reference voltage which is ideally in phase with the input signal, such that the multiplication factor is +1 during the positive half-cycle of the input and -1 during the negative half-cycle. It then functions as a perfect rectifier with the DC component of its output equal the mean value of the rectified input sine wave. If the input amplitude varies at some angular frequency $f_{\mathrm{s}}\left(<<f_{\mathrm{b}}\right)$ due to modulation of the radiant power on the detector, then the demodulator DC output will also vary accordingly.

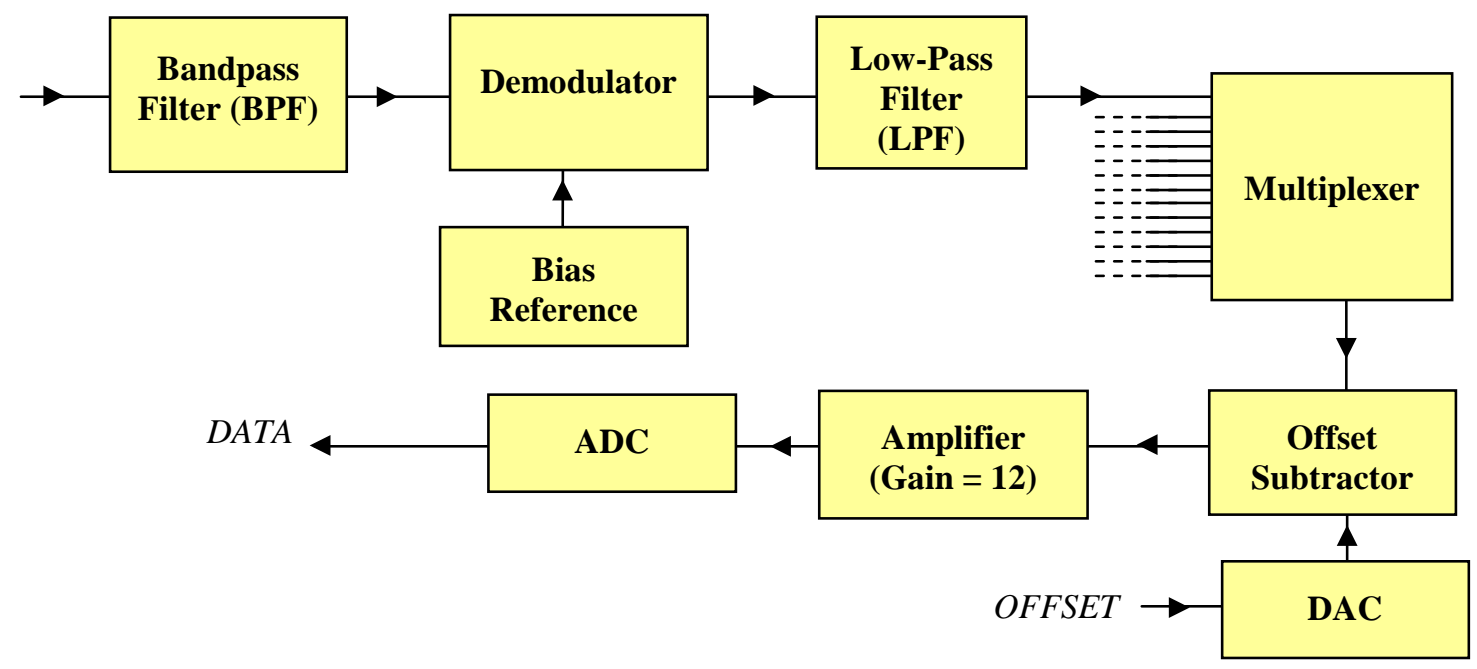

Figure 2: SPIRE on-board electronics signal chain.

Low-pass filter (LPF): The low pass filter following the demodulator is a 4-pole Bessel filter with $5 \mathrm{~Hz}$ 3-dB frequency, and rejects higher-frequency components from the demodulator output, passing just the slowly varying component proportional to the detector voltage. The LPF gain vs. signal modulation frequency is shown in Figure 4.

Multiplexer and offset subtraction: The LIA outputs are first multiplexed in groups of 16 , and a second stage of multiplexing combines three groups of 16 to form a group of 48 channels. After multiplexing, the pre-determined DC offsets are subtracted for all the channels. At the start of each observation, the value of OFFSET is defined for each detector individually by the DCU according to an automatic procedure designed to ensure that the dynamic range requirements will be met during the observation.

Digitisation: After offset subtraction, the signal is further amplified by a gain of 12 and then digitised, producing a binary output value DATA, with range 0 to $\left(2^{16}-1\right)$, which is sent to the DPU for telemetry.

The time variation of the system output depends on the time variation of the radiant power on the bolometer and on overall the transient response of the system (bolometers, cryoharness, and low-pass filter). In the case of point source or jiggle-map observations, the BSM movements are completed within approx. $20 \mathrm{~ms}$, and a typical bolometer time constant is $6 \mathrm{~ms}$. The transient response is therefore dominated by the LPF, as illustrated in Figure 3, which shows a simulation of the response to BSM movements at $t=0$ and $t=250 \mathrm{~ms}$, corresponding to $2-\mathrm{Hz}$ chopping (the bolometer is here assumed to have no slow response component: $a=0$ ). The sampling rate in chopped mode is such that the onground demodulation of the chop can be based on three samples on each of the flat parts of the waveform.

In scan map mode, when the beam crosses a point source, a Gaussian variation of radiant power with time ensues. In the absence of a bolometer slow-response component, the response of the signal chain to this input is again dominated by the LPF. For the nominal scan speed of 30 " $\mathrm{s}^{-1}$, the effect is to delay the output by approx. $74 \mathrm{~ms}$, with minimal $(<2 \%)$ distortion of the Gaussian profile, as shown in Figure 5a But if there is an appreciable bolometer slow-response component, then the effect is to diminish and distort the time-line with a long tail as the slow response persists following the peak. This is illustrated in Figure $5 \mathrm{~b}$ for the case of $\tau_{2}=0.5 \mathrm{~s}$, and $a=0.2$. 


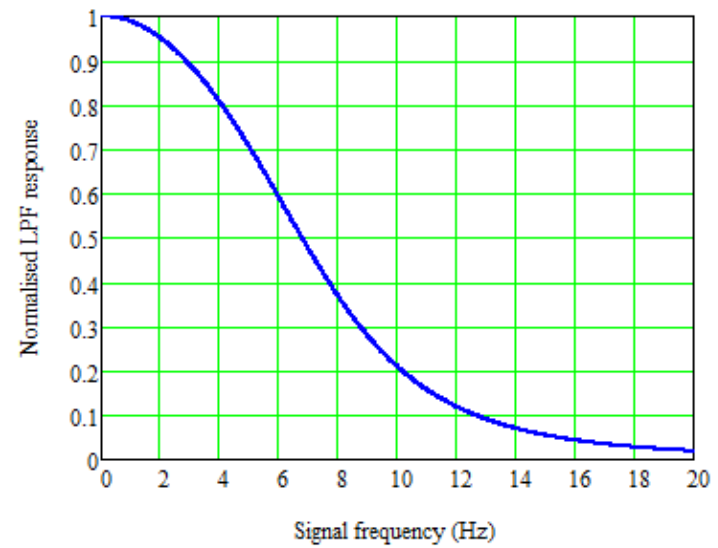

Figure 4: Low-pass filter gain vs. signal frequency.

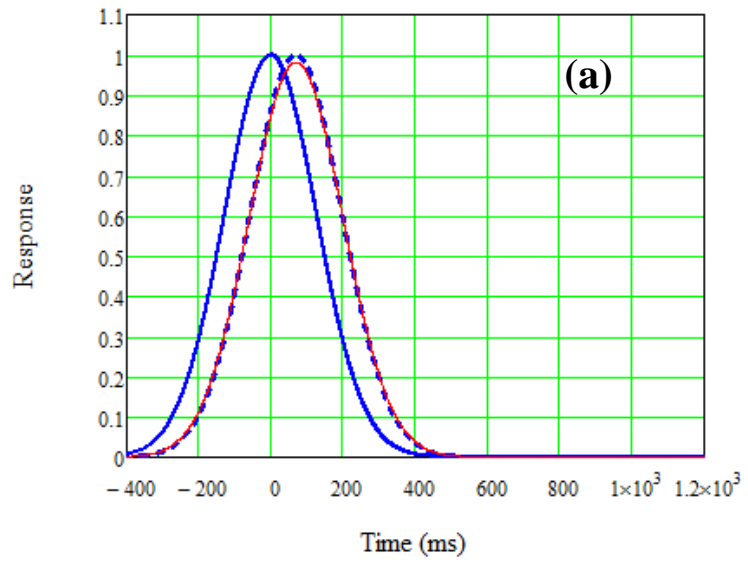

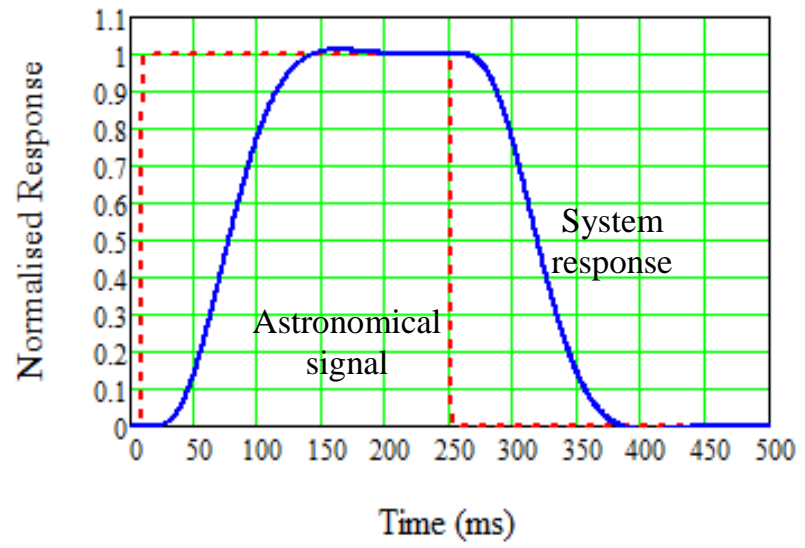

Figure 3: Overall signal chain response for a $250 \mu \mathrm{m}$ point source observation with BSM movements starting at $t=0$ and $250 \mathrm{~ms}$ (2-Hz chop frequency). A pure first-order bolometer response is assumed (i.e., $a=0$ ).

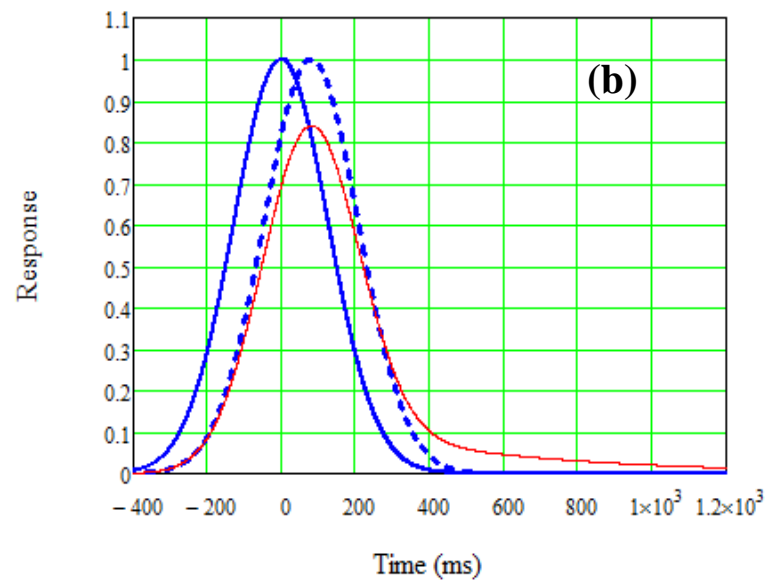

Figure 5: (a) Simulated signal chain response (thin solid line) to an 18 " FWHM, 60" $\mathrm{s}^{-1}$ scan rate astronomical point source timeline centred at $t=0$ (thick solid line) for a pure first-order detector response $(a=0)$ with $\tau_{1}=6 \mathrm{~ms}$. The dotted curve shows the astronomical timeline delayed by $74 \mathrm{~ms}$. (b) Response (thin solid line) to the same input for, $\tau_{1}=$ $6 \mathrm{~ms}, \tau_{2}=0.5 \mathrm{~s}, a=0.2$. The dotted curve is the astronomical timeline delayed by $80 \mathrm{~ms}$.

\section{SCAN-MAP PIPELINE}

The measured bolometer voltage can be taken to have two contributions, one dictated by the combination of the bias voltage and the background loading from the telescope, and the other due to the astronomical sky. Let the measured bolometer rms voltage be

$$
V_{\mathrm{d}-\mathrm{RMS}}=V_{\mathrm{o}}+V_{\mathrm{S}} \text {, }
$$

where $V_{\mathrm{o}}$ is the operating point voltage under identical operating conditions but in the absence of any astronomical signal, and $V_{\mathrm{S}}$ is due to the astronomical signal ( $V_{\mathrm{S}}$ is actually negative as the bolometer resistance decreases with increasing absorbed power). The behaviour of the operating point voltage as the photometric background on the bolometer is changed is illustrated in Figure 6 which shows the form of the load curve under different conditions. $V_{\mathrm{o}}$ depends on the bolometer parameters, operating temperature, and bias setting, and on the telescope background power. 
$V_{\mathrm{S}}$ is linearly related to the source flux density for small signals, but there is a departure from linearity for large signals. If $V_{\mathrm{o}}$ were known precisely, it could be subtracted to allow a calculation of source flux density by the pipeline with no systematic offset. However, this is regarded as too ambitious, at least at this stage, because variations of $V_{\mathrm{o}}$ (with telescope background, instrument temperature and detector temperature) may be difficult to track. Therefore, a constant value will be assigned to $V_{\mathrm{o}}$ (for a given detector with a given bias setting). $V_{\mathrm{o}}$ values will be determined by a blank sky observation under the nominal operating conditions, and should thus be close to the ideal values.

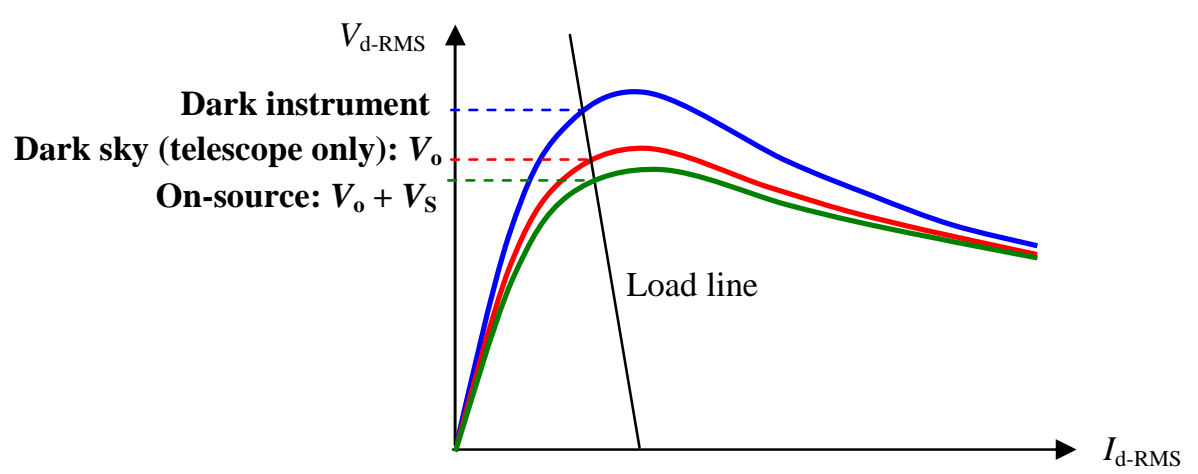

Figure 6: Dependence of operating point voltage on the operating conditions. $V_{\mathrm{o}}$ is the operating point voltage when viewing blank sky, and $V_{\mathrm{S}}$ is the change from this voltage introduced by the source.

The scan-map pipeline is shown in block diagram form Figure 7. The first step is to calculate the bolometer rms voltage. Based on knowledge of the electronics characteristics and the adopted bias frequency, the voltage is computed from the telemetered data and offset values using an iterative process that corrects for the $R C$ time constant formed by the bolometer-JFET harness (which depends on $R_{\mathrm{b}}$ ). The next three modules (first-level deglitching, removal of electrical crosstalk, and correction for the electrical filter) are applied to the voltage timelines as they can be regarded as electrical effects. The timelines are then converted to units of astronomical flux density, a process which includes subtraction of the fixed offset voltage, $V_{\mathrm{o}}$, flat fielding, and correction for any non-linear response to strong astronomical signals.

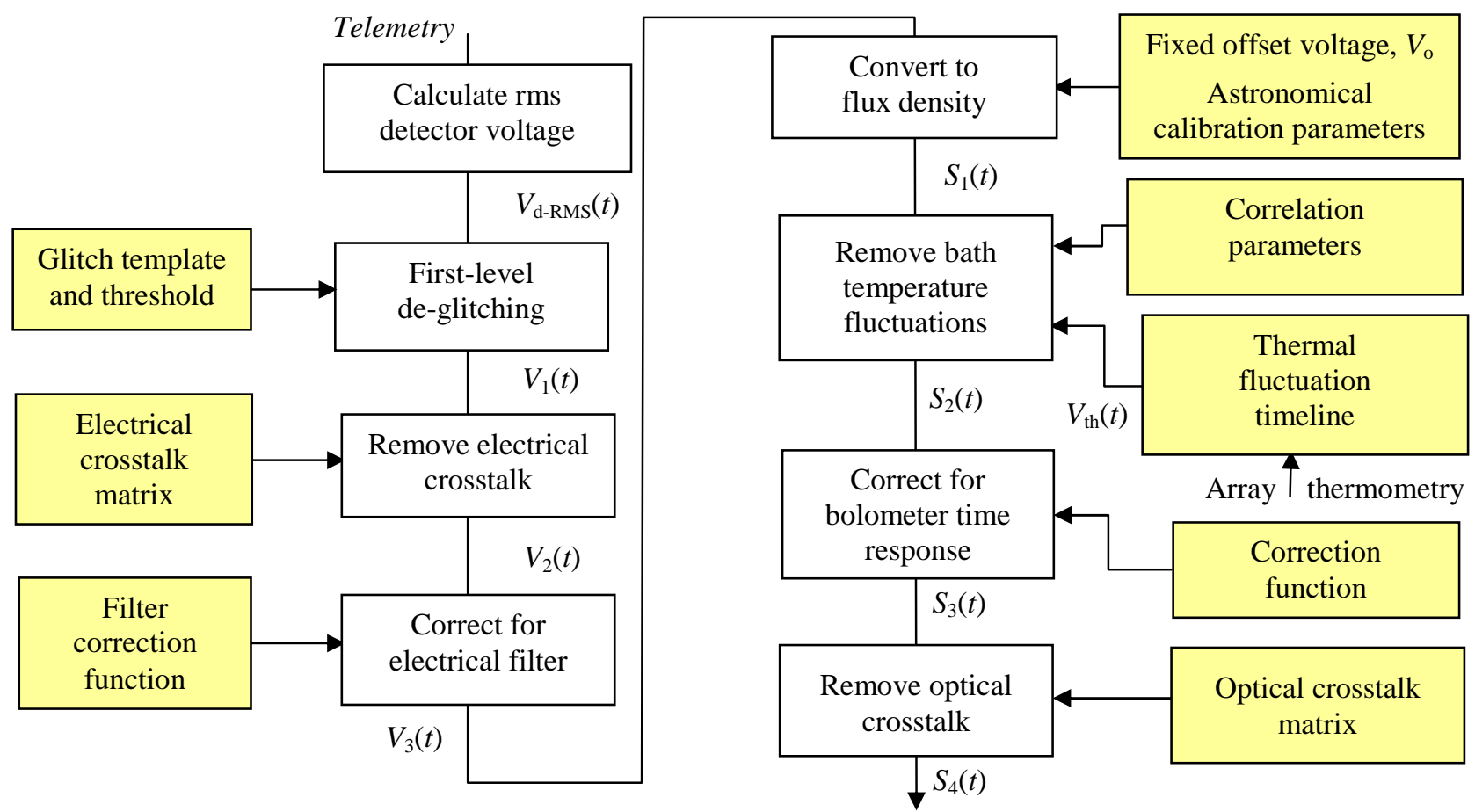

Figure 7: Pipeline block diagram for scan-map observations. 
Corrections are then made for other effects (bath temperature fluctuations, bolometer response, and optical crosstalk) which are linear when applied in terms of the power absorbed by the bolometer (which is proportional to flux density).

First-level deglitching: Before further processing of the measured detector voltage timeline, $V_{\mathrm{d}-\mathrm{rms}}(t)$, glitches due to cosmic ray hits or other impulse-like events in the detectors is removed by a method involving a wavelet-based local regularity analysis ${ }^{6}$. This process has two steps: the first detects glitch signatures over the measured signal and the second step locally reconstructs a signal free of such glitch signatures. Glitches are detected in the input timeline by wavelet analysis assuming that the glitch signature is similar to the signature of an impulse. The localized wavelet coefficients associated with a specific glitch are removed and a local, inverse wavelet transform is performed to create the de-glitched timeline. The output of this module is the deglitched voltage timeline is $V_{1}(t)$.

Removal of electrical crosstalk: The deglitched timeline may contain contributions that depend on the signals from other detectors due to either electrical or optical crosstalk. Electrical crosstalk arises after the detector and is due to capacitative or inductive coupling between the detector readout channels. Optical crosstalk occurs before the detector and is due to diffraction or aberrations in the optical system causing some of the power from an astronomical source to fall on inappropriate detectors. Electrical crosstalk can be removed if the coupling between the detectors is known, and it is appropriate to do it at this stage. The removal of optical crosstalk is done after the conversion to flux density (see below). We assume that (i) electrical crosstalk is linear, so that the effects can be characterised by a crossstalk matrix with constant elements; (ii) crosstalk from one detector to another involves negligible diminution of the signal in the primary detector, so that the diagonal elements of the crosstalk matrix are unity; (iii) there is no crosstalk between different arrays. For a particular time-step, the vector of electrical crosstalk-corrected signals is given by a simple matrix multiplication:

$$
\mathbf{V}_{2}=\mathbf{C}_{\text {elec }} \mathbf{V}_{1}
$$

Determination of the electrical crosstalk matrix elements in flight will be carried out using ionising radiation hits that the bolometers will experience. Ideally, a single event in a bolometer produces a spike only in its own output; crosstalk results in this being accompanied by lower-level responses from other detectors. In the absence of crosstalk, or if the crosstalk correction is to be left out, then the non-diagonal coefficients of $\mathbf{C}_{\text {elec }}$ are set to zero. The crosstalk corrected timeline for detector $\mathrm{i}$ is denoted $V_{2}(\mathrm{t})$.

Correction for LPF response: As noted in Section 3, the LPF imposes a delay on the detector data with respect to the telescope position along the scan, and this must be corrected to match the pointing timeline to the detector timeline. This is done by Fourier transforming the detector timeline, multiplying the FT by a correction function (based on the LPF transfer function) and transforming back to the time domain to obtain the corrected signal voltage, $V_{3}(t)$. The correction function, is derived from the LPF parameters (nominally the same for all detectors). To a close approximation, the overall effect of this step is to shift the data timeline by $74 \mathrm{~ms}$ with respect to the pointing timeline.

Conversion to flux density: The conversion also incorporates flat fielding and strong source corrections. For NTD germanium bolometers, whose behaviour is accurately described by an ideal bolometer model ${ }^{7,8}$, the small-signal responsivity for a given applied bias voltage varies with the voltage across the bolometer with a near-linear relationship over a wide range of background loading and bath temperature conditions. This translates to a corresponding relationship for the differential sensitivity of the system to flux density: writing $V_{\mathrm{d}-\mathrm{RmS}}$ as $V$, we have $\mathrm{d} V / \mathrm{d} S \propto V$. To allow for the fact that the responsivity-operating point voltage relationship will not be exactly linear, we let

$$
\frac{\mathrm{d} S}{\mathrm{~d} V}=f(V)
$$

We find that an accurate fit to the shape of $f(\mathrm{~V})$ an be made using a function of the form:

$$
f(V)=K_{1}+\frac{K_{2}}{V-K_{3}}
$$

where $K_{1}, K_{2}$ and $K_{3}$ are constants. $K_{3}$ and the ratio $K_{1} / K_{2}$ can be found by pointing the telescope at a selection of bright sources (without chopping) and measuring the relative change in responsivity as a function of bolometer voltage using the internal calibration source $\mathrm{PCal}^{9}$. It is not necessary to know how bright these sources are - they are just being used 
to establish a range of backgrounds on the detectors. The absolute values of $K_{1}$ and $K_{2}$ can be determined by observation of a single suitable astronomical calibrator.

A flux density corresponding to the measured rms detector voltage, $V_{3}$, can be derived by integrating the above expression between some fixed detector voltage, $V_{\mathrm{o}}$, and $V_{3}$ :

$$
S_{1}=\int_{V_{\mathrm{o}}}^{V_{3}}\left(K_{1}+\frac{K_{2}}{V-K_{3}}\right) \mathrm{d} V=K_{1}\left(V_{3}-V_{\mathrm{o}}\right)+K_{2} \ln \left(\frac{V_{3}-K_{3}}{V_{\mathrm{o}}-K_{3}}\right) .
$$

Ideally, $V_{\mathrm{o}}$ would be the bolometer voltage in the absence of any astronomical signal (i.e., what would be measured when observing blank sky in otherwise identical conditions). The resulting flux density would correspond to that from the sky calibrated with respect to the dark sky level. $V_{\mathrm{o}}$ will therefore be derived from standard calibration observations of a "dark" area of sky in scan-map mode, to determine the offset voltages for the detectors under the nominal conditions: bias voltage and frequency, detector and FPU temperature, and telescope temperature. Although ideally the conditions would be the same for the calibration and science observations, small differences are likely in practice. We therefore expect that, even for dark sky, $V$ will differ from the ideal value $V_{\mathrm{o}}$ (by an amount much larger than most astronomical signals). This means that the initial flux density values produced in this step will include offsets that must be removed later to derive the flux density from the sky. This is done naturally as part of the map-making routine.

The output of this module is a set of timelines corresponding to the first estimates of flux densities, $S_{1}(t)$.

Removal of correlated noise due to bath temperature fluctuations: Any ${ }^{3} \mathrm{He}$ bath temperature $\left(T_{\mathrm{o}}\right)$ fluctuations will influence all detectors in an array coherently - the temperature and corresponding output voltages will go up and down in synchronism. For the low-level fluctuations expected, the main effect of $T_{\mathrm{o}}$ variations will be the direct response of the detector output voltage. There will be a small second-order effect on the detector small-signal responsivity. Fluctuations in $T_{\mathrm{o}}$ are expected to be much slower than the nominal chopping frequency of $2 \mathrm{~Hz}$, so that the correction will only be needed for scan-map observations. The procedure is as follows: a thermal fluctuation timeline $V_{\mathrm{th}}(t)$ is available for each array from a sensitive thermometer attached to the array. This is scaled by a pre-determined empirical correlation coefficient for each detector, and subtracted from that detector's signal timeline. To avoid introducing additional noise, the thermometer timeline must be significantly less noisy than the bolometer signal, and is therefore averaged over a suitable period (typically $1 \mathrm{sec}$. or more) - thermal fluctuations on timescales shorter than this will not be tracked. The correction scheme, and the results of its application to instrument test data are described in Schulz et al. ${ }^{10}$ The output of this module is a set of timelines of astronomical flux densities corrected for low-frequency thermal drifts: $S_{2}(t)$.

Correction for bolometer time response: The bolometers are assumed to behave according to the two-component bolometer transfer function described in Section 2.1. The procedure for correcting the timelines is essentially the same as for the electrical filter correction: the timeline is Fourier transformed, multiplied by a correction function (based on the parameters $\tau_{1}, \tau_{2}$, and $a$ ), and then transformed back to the time domain to obtain the corrected timeline. The output of this module is a set of timelines of astronomical flux densities corrected for the bolometer time response: $S_{3}(t)$.

Removal of optical crosstalk: Optical crosstalk is here defined as power from the astronomical sky that should be incident on one detector actually falling on another. It is important to note that in the case of SPIRE, neighbouring detectors are separated by an angle of $2 \lambda / D$ on the sky, and even if a source is on-axis for a given pixel, a small fraction of the source power will be incident on the neighbouring detectors due to telescope diffraction. Non-neighbouring detectors are sufficiently far apart that they should not pick up any power from an on-axis source. Optical crosstalk can be characterised by a crosstalk matrix, $\mathbf{C}_{\text {opt }}$, analogous to the electrical crosstalk matrix described above. The vector of optical crosstalk-corrected flux densities is then given by

$$
\mathbf{S}_{4}=\mathbf{C}_{\text {opt }} \mathbf{S}_{3} .
$$

Unlike the case of electrical crosstalk, the diagonal elements are not equal to unity since optical crosstalk involves loss of power from the primary detector. The optical crosstalk matrix coefficients must be determined from calibration observations involving scanning a strong point source across each of the detectors in the array. In the absence of optical crosstalk, or if the crosstalk correction is to be left out, then the non-diagonal coefficients are set to zero and the diagonal coefficients are set to unity. The output of this module is a set of flux density timelines, $S_{4}(t)$, suitable for input to the map-making module. 
Map-making: SPIRE will use an implementation of the maximum likelihood map-making algorithm MADmap ${ }^{11}$, which was chosen following a systematic evaluation of several map-making codes using simulated SPIRE data ${ }^{12}$. This type of algorithm makes use of the redundant information from cross-linked observations to establish a noise covariance matrix which is then used to down-weight the contribution from $1 / f$ noise to the map ${ }^{13}$, and is widely used by cosmic microwave background (CMB) experimenters ${ }^{14}$. Greater levels of cross-linking enable a maximum likelihood map-maker to operate more effectively. As a result, this method is dependent on the observing strategy used. If cross-linked observations are not performed, then the output from this algorithm will be a naïve map (i.e. a map in which each pixel value is equal to the average value of all of the measurements falling within the pixel area) as this is the optimal map reconstruction for data obtained via a non-cross-linked scanning strategy.

\section{POINT SOURCE AND JIGGLE-MAP PIPELINE}

There are some key differences between chopped photometric observation and scan map observations: (i) for chopped observations, there is no need to correct for low-frequency noise temperature fluctuations: these will be at frequencies less than the $2-\mathrm{Hz}$ chop frequency and so chopped out; (ii) chopped observations produce differential data, and the source flux density is measured with respect to the sky background in the vicinity of the source; (iii) the ambient background power in the two chop positions will be slightly different, producing a significant offset signal: the purpose of nodding is to subtract out this offset. The principles of chopping and nodding are illustrated in Figure 8a, which shows left $(\mathrm{L})$ and right $(\mathrm{R})$ beams in two nod positions designated A and $\mathrm{B}$.

(a)
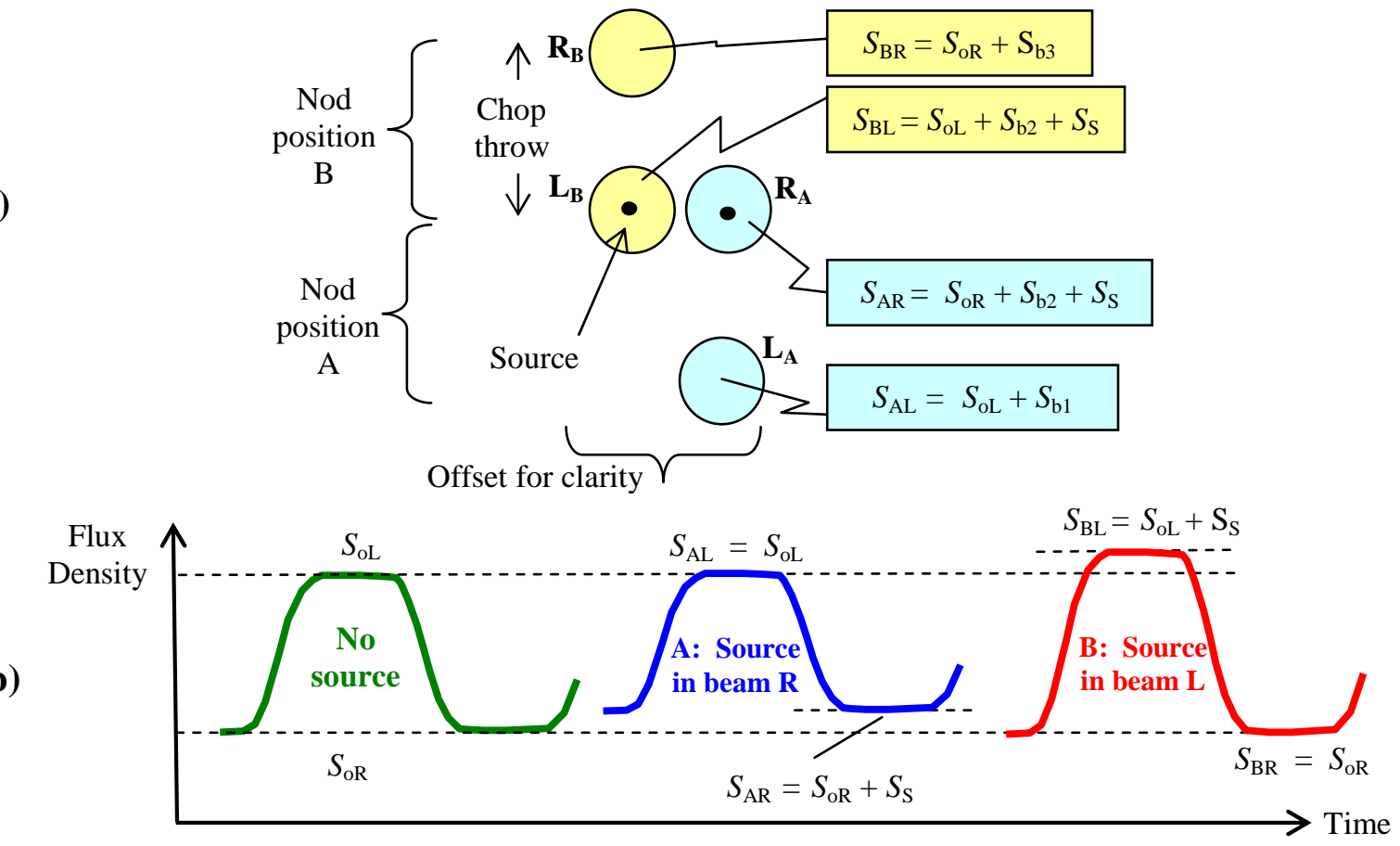

Figure 8: (a) Flux density levels measured during chopping and nodding. (b) Example timelines for nod positions A and B (with the source in the right beam for position A), where for simplicity the sky background is taken to be uniform.

Let $S_{\mathrm{oR}}$ and $S_{\mathrm{oL}}$ be the flux densities measured in the right and left beams for blank sky. Let $S_{\mathrm{b} 1}, S_{\mathrm{b} 2}$ and $S_{\mathrm{b} 3}$ be the sky background levels in the three positions, and let $S_{\mathrm{S}}$ be source flux density. The de-modulated chopped signal $(\mathrm{R}-\mathrm{L})$ for nod position A is $S_{\mathrm{A}}=\left(S_{\mathrm{oR}}+S_{\mathrm{b} 2}+S_{\mathrm{S}}\right)-\left(S_{\mathrm{oL}}+S_{\mathrm{b} 1}\right)$, whilst that for position B is $S_{\mathrm{B}}=\left(S_{\mathrm{oR}}+S_{\mathrm{b} 3}\right)-\left(S_{\mathrm{oL}}+S_{\mathrm{b} 2}+S_{\mathrm{S}}\right)$. The difference (de-modulated nod signal) is this $S_{\mathrm{A}}-S_{\mathrm{B}}=2 S_{\mathrm{S}}+\left(S_{\mathrm{b} 2}-S_{\mathrm{b} 1}\right)-\left(S_{\mathrm{b} 3}-S_{\mathrm{b} 2}\right)$. Thus, if the sky background is uniform or varying linearly, it is removed. If there is a higher order variation in sky brightness, then it will not be completely subtracted. 
Figure 8b illustrates chopped signal waveforms (for zero sky background) for the case of no source, source in beam $\mathrm{R}$ and source in beam L. Most of the difference between the chop positions is due to the asymmetric telescope background. In nod position A, the source increases the magnitude of the difference and in nod position B it decreases it.

Figure 9 shows the sequence of steps in the point source and jiggle-map pipeline, and the individual modules are described below.

First-level deglitching: As in the scan-map pipeline, before further processing of the measured detector voltage timeline, glitches will be removed. But the detector timelines in jiggle-map mode legitimately contain large changes between contiguous samples in the case of chopping on a strong source. Instead of the wavelet-based approach used for scan-map a simpler de-glitching scheme, involving rejection of outliers above a pre-defined threshold, is adopted. The output of this module is the de-glitched voltage timeline, $V_{1}(t)$.

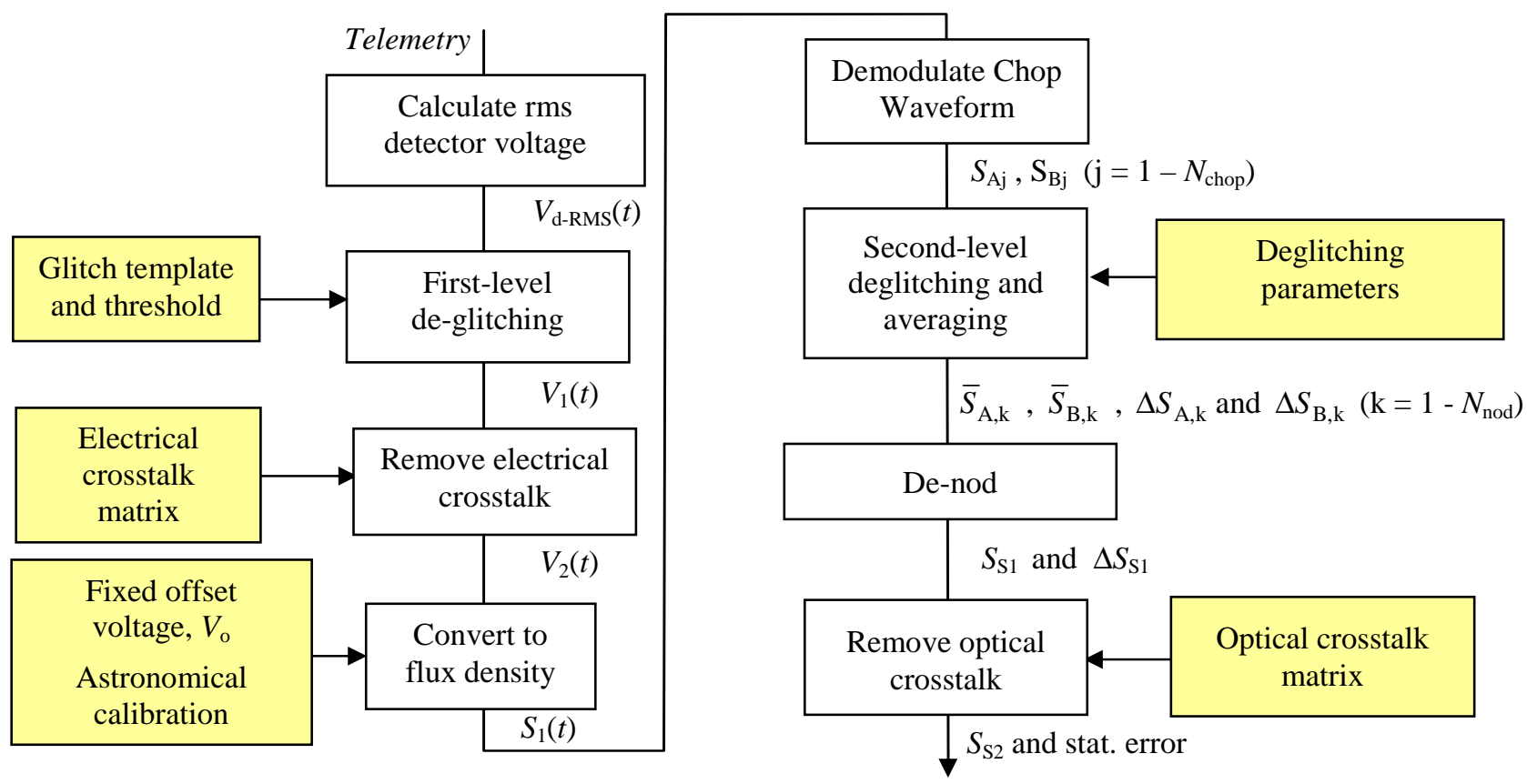

Figure 9: Pipeline block diagram for chopped/nodded observations at a single jiggle position, with $N_{\text {chop }}$ chop cycles per jiggle position and $N_{\text {nod }}$ nod cycles.

Removal of electrical crosstalk: This module is the same as in the scan-map pipeline.

Conversion to flux density: The pipeline must operate over a wide range of source brightness, and the direct proportionality of voltage to flux density cannot be assumed. It is therefore necessary to carry out the demodulation process after conversion to flux density to ensure that the subtraction in the subsequent de-nodding step is done in the linear regime. The voltage timelines $V_{2}(t)$ are converted to flux density timelines using the same module as for the scanmap pipeline (described in Section 4). The reference voltage $V_{\mathrm{o}}$ and the $K$-values are identical. The output of this module is a set of flux density timelines: $S_{1}(t)$.

Demodulation: The demodulated flux densities for nod positions A and B are calculated as:

$$
S_{\mathrm{A}}=\bar{S}_{\mathrm{AR}}-\bar{S}_{\mathrm{AL}} \quad \text { and } \quad S_{\mathrm{B}}=\bar{S}_{\mathrm{BR}}-\bar{S}_{\mathrm{BL}} .
$$

where $\bar{S}$ is the average of data for the relevant half-cycle, as explained in Section 3. For a given jiggle position with $N_{\text {chop }}$ chop cycles, the output of this module is thus $N_{\text {chop }}$ estimates of both $S_{\mathrm{A}}$ and $S_{\mathrm{B}}$ for each nod cycle: $S_{\mathrm{Aj}, \mathrm{k}}$ and $S_{\mathrm{Bj}, \mathrm{k}}$ where $\mathrm{j}=1-N_{\text {chop }}$ and $\mathrm{k}=1-N_{\text {nod. }}$. Note that for most practical cases $N_{\text {nod }}=1$. 
Second-level deglitching and averaging: For each nod cycle, the $N_{\text {chop }}$ estimates of the demodulated flux densities in each of the two nod positions can now be deglitched by rejecting outliers and averaging the remaining samples, to produce mean values and an associated uncertainty. The outputs of this module are, for each nod cycle, values of flux density and their associated uncertainties: $\bar{S}_{\mathrm{A}, \mathrm{k}} \pm \Delta S_{\mathrm{A}, \mathrm{k}}$ and $\bar{S}_{\mathrm{B}, \mathrm{k}} \pm \Delta S_{\mathrm{B}, \mathrm{k}}$.

De-nod: The de-nod process merely takes the difference between the flux densities in the two nod positions to derive the first estimate of the source flux density. For each of the $N_{\text {nod }}$ nod cycles, we have:

$$
S_{\mathrm{S}, \mathrm{k}}=\frac{1}{2}\left(\bar{S}_{\mathrm{A}, \mathrm{k}}-\bar{S}_{\mathrm{B}, \mathrm{k}}\right) \quad \text { with } \quad \Delta S_{\mathrm{S}, \mathrm{k}}=\frac{1}{2}\left(\Delta S_{\mathrm{A}, \mathrm{k}}^{2}+\Delta S_{\mathrm{B}, \mathrm{k}}^{2}\right)^{1 / 2} .
$$

If $N_{\text {nod }}>1$, then a weighted mean of the separate estimates of $\mathrm{S}_{\mathrm{S} 1}$ is be calculated as the final value. The final output of this module is a value of in-beam source flux density, $S_{\mathrm{S} 1}$, with an associated statistical uncertainty for each jiggle position of each detector.

Removal of optical crosstalk: Optical crosstalk is removed using the same method described in Section 4 to produce a final estimate, $S_{\mathrm{S} 2}$, of the in-beam source flux density and its associated statistical uncertainty. Note that the crosstalk matrix elements may be different to those in the case of the scan map pipeline due to the different BSM positions.

Mapmaking: For jiggle-map data, the next pipeline step is mapmaking, which is again carried out using MADmap (in this case a simple naïve map is all that is required).

Point source flux density derivation: A point source photometry observation produces, for each of the prime (onsource) detectors, flux density estimates for eight points (central position observed twice and six offset positions once each). A weighted fit of the eight data points is made to a 2-D Gaussian representation of the beam profile, assuming a point-like source, with the free parameters being peak flux density and two orthogonal positional offsets. The results are fitted flux density and offset and their associated uncertainties. The quality of the positional offset fit will be highly sensitive to the $\mathrm{S} / \mathrm{N}$. A point source observation also produces a sparse map of the background around the source, and the other detector data will also be processed to characterise this background.

\section{ASTRONOMICAL CALIBRATION}

The $K$-parameters used in the flux density conversion incorporate the astronomical calibration of the data. As noted in Section 4, this can be based on observations of a single primary calibration source. Uranus and Neptune have been chosen as the most suitable objects for this purpose - they are compact, moderately bright, and have well-understood brightness temperature spectra in the far infrared and submm ${ }^{15,16}$. Neptune is preferred as it is less bright, involving minimal non-linearity correction. In case the planets are not available for observation at the beginning of the mission, bright asteroids ${ }^{17,18}$ can be used, and a sample of faint stars has also been identified based on model stellar atmosphere spectra $^{19}$. It is planned to observe all three kinds of calibrators regularly throughout the mission in order to reduce uncertainties and establish a consistent calibration scheme covering a large brightness range and different source types.

The photometer pipeline calculates the flux densities at reference wavelengths of 250,350 and $500 \mu \mathrm{m}$. Because the pipeline processing cannot pre-judge the shape of the source spectrum over the photometric passband, a nominal spectrum must be assumed. The convention to be adopted by SPIRE and PACS is to assume a flat spectral energy distribution, i.e., $v S(v)$ constant across the band (corresponding to a spectral index of -1 ). The flux densities calculated by the pipeline can easily be converted to any other assumed source spectrum. If a power law spectrum is assumed, this is done by a straightforward multiplicative correction factor corresponding to the chosen spectral index. Typical SPIRE sources will have spectral index in the range $0-3$, requiring corrections up to a few $\%^{4}$. 


\section{REFERENCES}

1. M. Griffin, B. Swinyard, L. Vigroux, A. Abergel, P. Ade, P. André, J.-P. Baluteau, J. Bock, A. Franceschini, W. Gear, J. Glenn, M. Huang, D. Griffin, K. King, E. Lellouch, D. Naylor, S. Oliver, G. Olofsson, I. Perez-Fournon, M. Page, M. Rowan-Robinson, P. Saraceno, E. Sawyer, G. Wright, A. Zavagno, A. Abreu, G. Bendo, A. Dowell, C. D. Dowell, M. Ferlet, T. Fulton, P. Hargrave, G. Laurent, S. Leeks, T. Lim, N. Lu, H. Nguyen, A. Pearce, E. Polehampton, D. Rizzo, B. Schulz, S. Sidher, D. Smith, L. Spencer, I. Valtchanov, A. Woodcraft, K. Xu, L. Zhang, and the SPIRE Consortium, "Herschel-SPIRE: Design, Ground Test Results, and Predicted Performance", ", in Space Telescopes and Instrumentation I: Optical, Infrared, and Millimeter Wave, Marseille, 23-28 June 2008, Proc. SPIE 7010-02 (this volume).

2. G. Pilbratt, "Herschel mission overview and key programmes", in Space Telescopes and Instrumentation I: Optical, Infrared, and Millimeter Wave, Marseille, 23-28 June 2008, Proc. SPIE 7010-01 (this volume).

3. T.R. Fulton, D.A. Naylor, J.-P. Baluteau, M. Griffin, P. Davis-Imhof, B.M. Swinyard, T. Lim, C. Ordenovic, C. Surace, D. Clements, P. Panuzzo, R. Gastaud, E.T. Polehampton, and S. Guest, "The data processing pipeline for the Herschel/SPIRE imaging Fourier transform spectrometer", ", in Space Telescopes and Instrumentation I: Optical, Infrared, and Millimeter Wave, Marseille, 23-28 June 2008, Proc. SPIE 7010-102 (this volume).

4. 'SPIRE Observers' Manual”, HERSCHEL-HSC-DOC-0789 (http://herschel.esac.esa.int/Documentation.shtml)

5. W.S. Holland, E.I. Robson, W. K. Gear, C.R. Cunningham, J.F. Lightfoot, T. Jenness, R.J. Ivison, J.A. Stevens, P.A.R. Ade, M.J. Griffin, W.D. Duncan, J.A. Murphy, and D.A. Naylor, "SCUBA: a common-user submillimetre camera operating on the James Clerk Maxwell Telescope", Mon. Not. R. Astron. Soc. 303, 659, 1999

6. C. Ordenovic, C. Surace, B. Torresani, and A. Llebaria, "Glitches detection and signal reconstruction using Holder and wavelet analysis", Proc. SPIE, 5909, 526, 2005.

7. J.C. Mather, "Bolometer noise, nonequilibrium theory", Applied Optics, 21, 1125, 1982.

8. R.V. Sudiwala, M.J. Griffin, and A.L. Woodcraft, "Thermal modelling and characterisation of semiconductor bolometers", Int. Journal of Infrared and Mm Waves, 23, 545, 2002.

9. P. Hargrave, J.J. Bock, C. Brockley-Blatt, J. Coker, L. Duband, A. Goizel, D.K. Griffin, and B.M. Swinyard, "The $300 \mathrm{mK}$ system for Herschel-SPIRE", in Millimeter and Submillimeter Detectors and Instrumentation for Astronomy III, , Orlando, 27-31 May 2006, Proc. SPIE 6275, 2006.

10. B. Schulz, J.J. Bock, N. Lu, H.T. Nguyen, C.K. Xu, L. Zhang, C.D. Dowell, M.J. Griffin, G.T. Laurent, T.L. Lim, and B.M. Swinyard, "Noise Performance of the Herschel-SPIRE Bolometers during instrument ground tests", in Millimeter and Submillimeter Detectors and Instrumentation for Astronomy, Marseille, 23-28 June 2008, Proc. SPIE 7020-74.

11. C. Cantalupo, "MADmap: A fast parallel maximum likelihood CMB map making code", http://crd.lbl.gov/ cmc/MADmap/doc/man/MADmap.html, 2002.

12. D. L. Clements, P. Chanial, G. J. Bendo, C. K. Xu, B. Schulz, T. Waskett, B. Sibthorpe, G. T. Laurent, "Mapmaking algorithm selection for Herschel Spectral and Photometric Imaging Receiver (SPIRE) scan maps", PASP (submitted).

13. M. Tegmark, "CMB mapping experiments: A designer's guide”, Phys. Rev.D, 56, 4514, 1997.

14. T. Poutanen, G. de Gasperis, E. Hivon, H. Kurki-Suonio, A. Balbi, J. Borrill, C. Cantalupo, O. Doré, E. Keihänen, C.R. Lawrence, D. Maino, P. Natoli, S. Prunet, R. Stompor, and R. Teyssier, "Comparison of map-making algorithms for CMB experiments", Astron. Astrophys. 449, 1311, 2006.

15. M.J. Griffin and G.S. Orton, "The near-millimeter brightness temperature spectra of Uranus and Neptune", Icarus, $105,537,1993$.

16. M. Burgdorf, G.S. Orton, G.R. Davis, S.D. Sidher, H. Feuchtgruber, M.J. Griffin, and B.M. Swinyard, "Neptune's far-infrared spectrum from the ISO long-wavelength and short-wavelength spectrometers", Icarus, 164, 244, 2003.

17. T.G. Müller and J.S.V Lagerros, "Asteroids as far-infrared photometric standards for ISOPHOT", Astron. Astrophys., 338, 340, 1998.

18. T.G. Müller, the Herschel Calibration Steering Group, and the ASTRO-F Calibration Team, "The asteroid preparatory programme for Herschel, ASTRO-F \& ALMA", Proc. The dusty and molecular universe: a prelude to Herschel and ALMA, 27-29 October 2004, Paris, ESA SP-577, 471, 2005.

19. L. Decin and K. Eriksson, "Theoretical model atmosphere spectra used for the calibration of infrared instruments", Astron. Astrophys., 472, 1041, 2007. 